\title{
Modelos para estimar el volumen comercial de Bursera simaruba (L.) Sarg. y de Metopium brownei (Jacq.) Urb. en Quintana Roo, México Models to determine the commercial volume of Bursera simaruba (L.) Sarg. and of Metopium brownei (Jacq.) Urb. in Quintana Roo, México
}

\author{
Jonathan Hernández Ramos ${ }^{1 *}$, Juan Carlos Tamarit Urias ${ }^{2}$, Adrián Hernández
} Ramos $^{3}$, Xavier García Cuevas ${ }^{1}$, Enrique Buendía Rodríguez ${ }^{4}$

\begin{abstract}
The state of Quintana Roo occupies the second national place in timber production of broadleaf species, of which Bursera simaruba and Metopium brownei are relevant, from the value of their wood and wide distribution. The functions that estimate the stem and commercial volume, in addition to the taper and the volume ratio are important in the elaboration of a forest management program. The aim of this study was to fit a nonlinear function that estimates trade volume for two species in central and southern Quintana Roo. A commercial volume model with random effects was fitted at the tree level, using the mensuration data from 188 and 133 trees for each species, respectively, and all the diameter categories found in these forest ecosystems were covered. The best combinations of inclusion of the random effects were chosen according to the maximum likelihood. The proposed equations have an average bias when estimating the commercial volume for the two species of $0.0045 \mathrm{~m} 3$ and an explanation greater than $90 \%$ of the sample variability. The resulting equations may be used in the timber estimation of the sustainable forest management programs of tropical forests at the central and southern territories of the state.
\end{abstract}

Key words: Tropical forest, product distribution, forest management, cubing system, Quintana Roo, volume.

\section{Resumen}

El estado de Quintana Roo ocupa el segundo lugar nacional en producción maderable de especies latifoliadas; de ellas, Bursera simaruba y Metopium brownei son de relevancia forestal debido al valor de su madera y amplia distribución en la entidad. Para elaborar programas de manejo forestal, las funciones que estiman el volumen fustal y comercial, además del ahusamiento y la razón de volumen son importantes. El objetivo del presente estudio fue ajustar una función no-lineal que estime el volumen comercial para dos taxa en el centro y sur de Quintana Roo, México. Con información dasométrica de 188 y 133 árboles, respectivamente que abarcaron todas las categorías diamétricas observadas en estos ecosistemas forestales; se ajustó un modelo de volumen comercial con efectos aleatorios a nivel de árbol, y se eligieron las mejores combinaciones de inclusión de los efectos aleatorios según la máxima verosimilitud. Las ecuaciones propuestas tienen un sesgo, en promedio, al estimar el volumen comercial para los dos taxones de $0.0045 \mathrm{~m} 3$ y una explicación mayor a $90 \%$ de la variabilidad muestral. Las ecuaciones resultantes podrán emplearse en la estimación maderable en los programas de manejo forestal sustentable de los bosques tropicales ubicados en el centro y sur del estado.

Palabras clave: Bosque tropical, distribución de productos, manejo forestal, sistema de cubicación, Quintana Roo, volumen.

Fecha de recepción/Reception date: 2 de julio de 2020

Fecha de aceptación/Acceptance date: 20 de septiembre de 2021.

\footnotetext{
${ }^{1}$ Instituto Nacional de Investigaciones Forestales, Agrícolas y Pecuarias. Campo Experimental Chetumal. México.

${ }^{2}$ Instituto Nacional de Investigaciones Forestales, Agrícolas y Pecuarias. Campo Experimental San Martinito. México.

${ }^{3}$ Instituto Nacional de Investigaciones Forestales, Agrícolas y Pecuarias. Campo Experimental Saltillo. México.

${ }^{4}$ Instituto Nacional de Investigaciones Forestales, Agrícolas y Pecuarias. Campo Experimental Valle de México. México.

*Autor para correspondencia; correo-e: forestjonathanhdez@gmail.com
} 


\section{Introducción}

El estado de Quintana Roo (Q. Roo) ocupa el segundo lugar nacional en producción maderable de taxones latifoliados, con una cosecha aproximada de $47139 \mathrm{~m}^{3}$ de madera en rollo $\left(\mathrm{m}^{3} \mathrm{mr}\right)$ de especies comunes tropicales $(12.10 \%$ de la producción total) y de $4807 \mathrm{~m}^{3} \mathrm{mr}$ de especies preciosas (15.18\% de la producción nacional), con un valor de aproxímadamente \$84 091064 y \$17 306 518, respectivamente, que lo ubica con una contribución al PIB por abajo de $0.2 \%$ del registrado a nivel nacional (Semarnat, 2017). Bursera simaruba (L.) Sarg. (chakáh) y Metopium brownei (Jacq.) Urb. (chechem negro) son especies comunes tropicales de interés comercial para la industria forestal (Silva et al., 2011; Gallegos et al., 2012), se distribuyen ampliamente en la Península de Yucatán (Herbario CICY, 2010), son utilizadas en la agroforestería por su crecimiento y regeneración (Bursera simaruba); así como para la elaboración de muebles y artesanías (Metopium brownei) (Román et al., 2016; OIMT, 2020).

La estimación precisa del volumen fustal, del volumen total al incluir las ramas, y del volumen comercial a partir de las dimensiones que exige el mercado es importante para desarrollar las prácticas silvícolas propuestas en el plan de manejo forestal, de acuerdo con los datos de los inventarios forestales realizados (CorralRivas y Návar-Cháidez, 2009; Diéguez-Aranda et al., 2009; Gómez-García et al., 2016; Tamarit et al., 2017; Niño et al., 2018).

La modelación matemática es una herramienta de apoyo para los manejadores de los recursos forestales, por lo que el ajuste de modelos que estiman el volumen fustal, total y comercial; o modelen, con precisión, la proporción entre el volumen comercial y el fustal $(V c / V f)$, así como su dinámica de disminución en el volumen acumulado de un árbol, con el incremento de la altura y la reducción del diámetro sobre el fuste, hasta llegar a la altura total (funciones de razón de volumen); además de que describan el ahusamiento de los árboles (diámetro a diferentes 
alturas) por especie contribuye a la realización de planes de manejo acordes a las condiciones específicas y actuales de los recursos (Burkhart, 1977; Tapia y Návar, 2011; Barrios et al., 2014; Özçelik y Göçeri, 2015; Hernández-Ramos et al., 2018a).

En México, durante años recientes, la modelación se ha realizado por medio de funciones que consideran los diámetros a distintas alturas sobre el fuste y sistemas compatibles de ahusamiento - volumen comercial para diferentes especies del género Pinus (Tapia y Návar, 2011; Quiñonez-Barraza et al., 2014; Tamarit et al., 2016; Silva et al., 2018); o por modelos de razón de volumen para especies como Eucalyptus urophylla Blake en Huimanguillo, Tabasco, Swietenia macrophylla King en Quintana Roo y P. pseudostrobus Lindl. en San Juan Nuevo Michoacán, entre otras (Hernández-Ramos et al., 2018a; García-Espinoza et al., 2018).

Los sistemas de volumen comercial compuestos por modelos de razón son una herramienta para calcular con precisión el volumen comercial y la distribución de productos maderables de árboles en pie (Prodan et al., 1997; Hernández-Ramos et al., 2018a; García-Espinoza et al., 2018). La estimación de estos volúmenes es fundamental en la proyección del aprovechamiento del recurso maderable y el rendimiento para la industria forestal, porque posibilitan la estimación del ingreso financiero del bosque a partir de datos de inventarios forestales, principalmente la distribución de los productos por unidad de superficie (Niño et al., 2018; Silva et al., 2018); y contribuyen, además, a evitar el sobre aprovechamiento, en general, de taxones con importancia comercial, no solo en bosques tropicales.

Para la zona de interés, Hemández-Ramos et al. (2018a y 2018b) ajustaron modelos de volumen total y generaron un sistema de volumen comercial a partir de modelos de razón, además de un sistema segmentado de ahusamiento (d) compatible con el volumen comercial $(V c)$ para Swietenia macrophylla (caoba) en bosques tropicales de Quintana Roo. López et al. (2019) ajustaron un sistema compatible de ecuaciones de volumen comercial que consta de fuste, ramas y tallo y ramas gruesas de árboles para 11 especies tropicales importantes en el sureste de México. 
Tradicionalmente, las ecuaciones para diseñar esos sistemas de volumen comercial se han ajustado mediante modelos de regresión lineal y no lineal que asumen normalidad, igualdad de varianzas e independencia de los errores (Carrero et al., 2008).

Sin embargo, la obtención de ecuaciones confiables para algunas variables es compleja, debido a la variabilidad que existe en estas relaciones por efecto de las diferentes formas de los fustes y condiciones en que se desarrolla cada individuo (Quiñonez et al., 2012). En la actualidad, el ajuste bajo el enfoque de modelos de efectos mixtos (MEM) es una opción viable para dicho tipo de estudios (Corral-Rivas et al., 2014), ya que su incorporación permite agrupar las fuentes de variabilidad y reducir la varianza del error (Seoane, 2014). La técnica de MEM incluye en su formulación parámetros fijos comunes a toda la población y parámetros aleatorios específicos de cada nivel de agrupación (Seoane, 2014). De ello, resultan estimadores más eficientes, precisos y confiables que los parámetros fijos del modelo; y por lo tanto, se pueden hacer estimaciones con mayor certidumbre de la variable de interés (Carrero et al., 2008). Por todo lo anterior, el objetivo del presente estudio fue ajustar una función no-lineal con efectos aleatorios que estime el volumen comercial para Bursera simaruba y Metopium brownei en el centro y sur de Quintana Roo, México.

\section{Materiales y Métodos}

El estudio se realizó en distintas áreas forestales de Quintana Roo, México, que se ubican en zonas con topoformas de lomeríos bajos y llanura rocosa, a una altitud de 20-180 m. El clima predominante es de tipo cálido subhúmedo (Aw), con temperatura media anual de $22{ }^{\circ} \mathrm{C}$ y una precipitación media de $1300 \mathrm{~mm}$ (Semarnat, 2014). 
A partir de recorridos en las áreas forestales del estado, se seleccionaron árboles con diámetro normal superior a $7.5 \mathrm{~cm}$, situados aleatoriamente en zonas no perturbadas del bosque tropical, donde se muestrearon 188 ejemplares de Bursera simaruba y 133 de Metopium brownei, los cuales abarcaron todas las categorías diamétricas presentes en estos ecosistemas forestales $(10-50$ y $10-60 \mathrm{~cm}$, respectivamente). De cada uno de ellos, se obtuvo información del diámetro del tocón $(d t)$, diámetro normal $(d)$, altura total $(H)$ y diámetros y alturas parciales sobre el fuste $\left(d_{i}\right.$ y $\left.h_{i}\right)$. La muestra se eligió con árboles monopódicos, sin presencia de daños mecánicos ni de plagas o enfermeda $\geq$ des, además de estar por lo menos a $20 \mathrm{~m}$ de distancia de los caminos, claros o de lugares con desmontes para evitar efectos de borde. La estadística descriptiva de las variables analizadas se muestra en el Cuadro 1.

Cuadro 1. Estadística descriptiva de la muestra de árboles de Bursera simaruba (L.) Sarg. y Metopium brownei (Jacq.) Urb.

\begin{tabular}{|c|c|c|c|c|c|c|c|c|c|c|}
\hline \multirow{3}{*}{ Estadísticos } & \multicolumn{10}{|c|}{ Especie } \\
\hline & \multicolumn{5}{|c|}{ Bursera simaruba } & \multicolumn{5}{|c|}{ Metopium brownei } \\
\hline & $\boldsymbol{d}$ & $\boldsymbol{H}$ & $v \boldsymbol{t}$ & $\boldsymbol{R}$ & $V c$ & $\boldsymbol{d}$ & $\boldsymbol{H}$ & $v t$ & $\boldsymbol{R}$ & $V c$ \\
\hline Promedio & 23.82 & 13.97 & 0.4938 & 0.44 & 0.2143 & 30.00 & 15.45 & 0.6820 & 0.46 & 0.3174 \\
\hline Error típico & 0.24 & 0.08 & 0.0110 & 0.01 & 0.0080 & 0.30 & 0.09 & 0.0140 & 0.01 & 0.0120 \\
\hline Desviación estándar & 9.21 & 3.19 & 0.4360 & 0.38 & 0.3200 & 10.13 & 3.11 & 0.4840 & 0.37 & 0.3950 \\
\hline Coeficiente de variación & 38.66 & 22.88 & 88.2900 & 87.05 & 149.2900 & 33.77 & 20.13 & 70.9600 & 81.52 & 124.4300 \\
\hline Varianza & 84.79 & 10.22 & 0.1900 & 0.14 & 0.1020 & 102.64 & 9.67 & 0.2350 & 0.14 & 0.1560 \\
\hline Curtosis & -0.50 & -0.60 & 1.5000 & -1.50 & 2.3000 & -0.60 & 0.50 & -0.1000 & -1.60 & 3.2000 \\
\hline Coeficiente de asimetría & 0.60 & -0.10 & 1.4000 & 0.50 & 2.6000 & 0.20 & -0.50 & 0.8000 & 0.40 & 1.9000 \\
\hline Mínimo & 7.00 & 5.57 & 0.0239 & 0.01 & 0.0013 & 9.40 & 4.63 & 0.0337 & 0.02 & 0.0028 \\
\hline Máximo & 49.80 & 21.80 & 2.1000 & 1.00 & 2.1000 & 57.30 & 23.10 & 2.3000 & 1.00 & 2.3000 \\
\hline
\end{tabular}

$d=$ Diámetro normal $(\mathrm{cm}) ; H=$ Altura total $(\mathrm{m}) ; v t=$ Volumen total $\left(\mathrm{m}^{3}\right) ; R=$ Razón de volumen $\left(\mathrm{m}^{3}\right) ; V c=$ Volumen comercial $\left(\mathrm{m}^{3}\right)$. 
Las mediciones de los diámetros (di) a diferentes alturas sobre el fuste $\left(h_{i}\right)$ de $0.3 \mathrm{~m}$, $0.6 \mathrm{~m}, 0.9 \mathrm{~m}, 1.3 \mathrm{~m}$ y $2.5 \mathrm{~m}$ se realizaron de forma directa con una forcípula graduada al milímetro; mientras que el resto de $h i$ y $d_{i}$ con un Telerelascopio de Bitterlich a lo largo del fuste, cuando fue posible desde los $2.5 \mathrm{~m}$ hasta la altura total $(H)$ o diámetro cero $\left(d_{0}\right)$. Para el cálculo del volumen fustal $(V f)$ se sumaron los volúmenes de las distintas secciones (trozas), mismas que se determinaron mediante la fórmula de Smalian y para el volumen de punta o última troza $(V p)$ con la fórmula del cono; la razón de volumen $(R)$ resultó del cociente entre el volumen a distintas secciones sobre el fuste $(V i)$ y el $V f$, para ello se utilizó la expresión: Vi/Vf (Balzarini et al., 2005; Hernández-Ramos et al., 2018a; García-Espinoza et al., 2018).

Como se señala en la Norma Oficial Mexicana NOM-152-SEMARNAT-2006, para cada especie se ajustó una función no-lineal (modelo general), la cual está conformada por dos componentes: el primero incluye una estructura matemática que corresponde a una ecuación de $V f\left(a_{0}+a_{1}\left(d^{2} H / 1000\right)\right.$, y el segundo es una ecuación referida a la razón existente entre los volúmenes de la secciones y la $R$ $\left(e^{\left(-(a 2)^{*}((d i / d) / 1000)\right.} e^{\left(a 3^{*}(d i / d)\right)}\right)$. Ecuaciones que al multiplicarse estiman el volumen comercial (VC) (Schabenberger y Pierce, 2002; Balzarini et al., 2005) (Cuadro 2). Este último, se define por las dimensiones que demande la industria del aserrío o los productos maderables que se tenga interés de estimar: largas dimensiones o primarios, cortas o secundarios y desperdicio o leña. Para las dos especies se probó el modelo general y el efecto aleatorio aditivo a cada combinación de los parámetros fijos, y resultaron ocho expresiones a partir del modelo general. 
Cuadro 2. Modelos de volumen comercial ajustado con efectos fijos y aleatorios para Bursera simaruba (L.) Sarg.y Metopium brownei (Jacq.) Urb.

\begin{tabular}{cc}
\hline Identificador & Estructura de los modelos \\
\hline General & $V c=\left[a_{0}+a_{1} \cdot\left(d^{2} \cdot H / 1000\right)\right] \cdot\left[e^{\left(-a_{2} \cdot\left(\frac{d_{i}}{d} / 1000\right)\right)} \cdot e^{a_{3} \cdot \frac{d_{i}}{d}}\right]$ \\
\hline 1 & $V c=\left[\left(a_{0}+u\right)+a_{1} \cdot\left(d^{2} \cdot H / 1000\right)\right] \cdot\left[e^{\left(\left(-a_{2}+u\right) \cdot\left(\frac{d_{i}}{d} / 1000\right)\right)} \cdot e^{a_{3} \cdot \frac{d_{i}}{d}}\right]$ \\
\hline 2 & $V c=\left[\left(a_{0}+u\right)+a_{1} \cdot\left(d^{2} \cdot H / 1000\right)\right] \cdot\left[e^{\left(-a_{2} \cdot\left(\frac{d_{i}}{d} / 1000\right)\right)} \cdot e^{\left(a_{3}+u\right) \cdot \frac{d_{i}}{d}}\right]$ \\
\hline 3 & $V c=\left[a_{0}+\left(a_{1}+u\right) \cdot\left(d^{2} \cdot H / 1000\right)\right] \cdot\left[e^{\left(\left(-a_{2}+u\right) \cdot\left(\frac{d_{i}}{d} / 1000\right)\right)} \cdot e^{a_{3} \cdot \frac{d_{i}}{d}}\right]$ \\
\hline $5 c=\left[a_{0}+\left(a_{1}+u\right) \cdot\left(d^{2} \cdot H / 1000\right)\right] \cdot\left[e^{\left(-a_{2} \cdot\left(\frac{d_{i}}{d} / 1000\right)\right)} \cdot e^{\left(a_{3}+u\right) \cdot \frac{d_{i}}{d}}\right]$ \\
\hline $6 c=\left[\left(a_{0}+u\right) \cdot\left(d^{2} \cdot H / 1000\right)^{a_{1}}\right] \cdot\left[e^{\left(\left(-a_{2}+u\right) \cdot\left(\frac{d_{i}}{d} / 1000\right)\right)} \cdot e^{a_{3} \cdot \frac{d_{i}}{d}}\right]$ \\
\hline $7 c=\left[\left(a_{0}+u\right) \cdot\left(d^{2} \cdot H / 1000\right)^{a_{1}}\right] \cdot\left[e^{\left(-a_{2} \cdot\left(\frac{d_{i}}{d} / 1000\right)\right)} \cdot e^{\left(a_{3}+u\right) \cdot \frac{d_{i}}{d}}\right]$ \\
\hline $6 c=\left[a_{0} \cdot\left(d^{2} \cdot H / 1000\right)^{\left(a_{1}+u\right)}\right] \cdot\left[e^{\left(\left(-a_{2}+u\right) \cdot\left(\frac{d_{i}}{d} / 1000\right)\right)} \cdot e^{a_{3} \cdot \frac{d_{i}}{d}}\right]$ \\
\hline $6 c=\left[a_{0} \cdot\left(d^{2} \cdot H / 1000\right)^{\left(a_{1}+u\right)}\right] \cdot\left[e^{\left(-a_{2} \cdot\left(\frac{d_{i}}{d} / 1000\right)\right)} \cdot e^{\left(a_{3}+u\right) \cdot \frac{d_{i}}{d}}\right]$
\end{tabular}

$d=$ Diámetro normal $(\mathrm{cm}) ; d_{i}=$ Diámetro a distintas alturas sobre el fuste $; H=$ Altura total $(\mathrm{m}) ; V c=$ Volumen comercial $; a_{0}, a_{1}, a_{2}$, y $a_{3}=$ Parámetros por ser estimados; $+u=$ Efecto aleatorio; $e=$ Función exponencial.

El modelo general (Cuadro 2) se ajustó, en principio, por mínimos cuadrados ordinarios (MCO) y dos variantes de la ecuación de volumen fustal de Spurr (1952): una lineal de la forma $y=a_{0}+a_{1} X$, y la segunda de tipo exponencial de forma $y=a X^{b}$. En ellas, $y$ es la variable respuesta ( $V f=$ volumen fustal), $X$ representa la variable independiente incluida en el modelo $\left(d^{2} H\right)$, a y $b$ corresponden a los parámetros por ser estimados.

La incorporación del efecto aleatorio a los parámetros fijos $\left(a_{i}+u\right)$ se hizo por árbol para explicar la variabilidad en su ahusamientos, al considerar el sentido 
biológico de cada uno; por ejemplo, al incluir el efecto aleatorio en el parámetro referente a la forma del árbol, la velocidad de cambio de cuerpo geométrico en el fuste, la razón de proporción entre el volumen fustal y el volumen comercial o en los tres de manera simultánea dentro de cada uno de los componentes en la función no-lineal propuesta, en la que el valor de probabilidad del efecto deberá ser significativo $(P \geq 0.05)$ (Cuadro $1,1-16)$.

El ajuste se realizó en el programa SAS $9.3^{\circledR}$ mediante el procedimiento nlmixed y el método de primer orden FIRO, el cual define con mayor precisión la aproximación por verosimilitud de los valores en los parámetros estimados (Beal y Sheiner, 1982; SAS, 2011). La distribución tanto para las variables dependientes, como para los efectos aleatorios $\left(a_{i}+u\right)$ se especificó en la programación como NORMAL para ajustarlos por la técnica de optimización Dual Cuasi-Newton y el método de integración Cuadratura Gaussiana Adaptativa del Sistema de Análisis Estadísticos (SAS, 2011).

La selección de la mejor combinación, cuando se incorporan los efectos aleatorios dentro del modelo no-lineal, se hizo a través de la significancia de los parámetros estimados tanto en efectos fijos, como aleatorios a un valor de $95 \%$ de confiabilidad $(P \geq 0.05$ ), y los mejores valores de Verosimilitud-2Log y Criterio de Información de Akaike Corregido (CIAC) (García y Rapelli, 2011; Gómez-García et al., 2016); además, para la mejor expresión se verificó de forma gráfica la tendencia en las estimaciones al emplear por separado las estructuras del modelo en el volumen fustal ( $V f=$ primera estructura matemática) y razón de volumen ( $R=$ segunda estructura matemática), y de manera conjunta para estimar el volumen comercial $(V c)$ de cada especie, o hasta un diámetro de $20 \mathrm{~cm}$ (Cuadro 1, modelo general).

En el modelo seleccionado se verificó la homocedasticidad de los residuos mediante la prueba de White, bajo las hipótesis de que los residuales se distribuyen de manera homogénea alrededor de cero (Hipótesis nula: Ho); mientras que, la hipótesis alternativa refiere una distribución heterocedástica de los residuos (Hipótesis alternativa: $\mathrm{Ha}$ ) a un 
nivel de confiabilidad de $95 \%$ (Martínez et al., 2006). La calidad de las estimaciones para las variables $V f, R$ y $V c$ se determinó a través del sesgo $(E)$ (Quevedo et al., 2010) y el coeficiente de determinación $\left(R^{2}\right)$ y con el análisis gráfico de los valores observados contra los valores estimados de cada variable (Pece, 1994).

\section{Resultados}

Al ajustar el modelo general y las variaciones en la inclusión de los efectos aleatorios, se observó que tanto la estructura general del modelo, como las expresiones 1, 2, 4 y 5 para Bursera simaruba; y 1, 2, 4 y 5 para Metopium brownei en algunos de sus parámetros fijos no fueron significativos a $95 \%$ de confiabilidad $(P \geq 0.05)$. Mientras que, en las combinaciones de inclusión de los efectos aleatorios de las expresiones 6 y 8 en ambas especies, los parámetros relacionados a los afectos mixtos no pudieron estimarse, aun cuando se trató de ajustar por el método Gauss-Hermite (Pinheiro y Bates, 1995). Por lo cual, se descartaron para posteriores análisis y no se presentan en el Cuadro 3. Debido a esa situación, solo se muestran las combinaciones de inclusión de los parámetros aleatorios de las expresiones 3 y 7 para árboles de Bursera simaruba y Metopium brownei para los análisis posteriores, en los cuales todos fueron significativos $(P \geq 0.05)$ al ajustarlos con 1476 y 1130 pares de datos, respectivamente. 
Cuadro 3. Parámetros estimados para las diferentes expresiones del modelo de volumen comercial ajustado para de Bursera simaruba (L.) Sarg.y Metopium brownei (Jacq.) Urb.

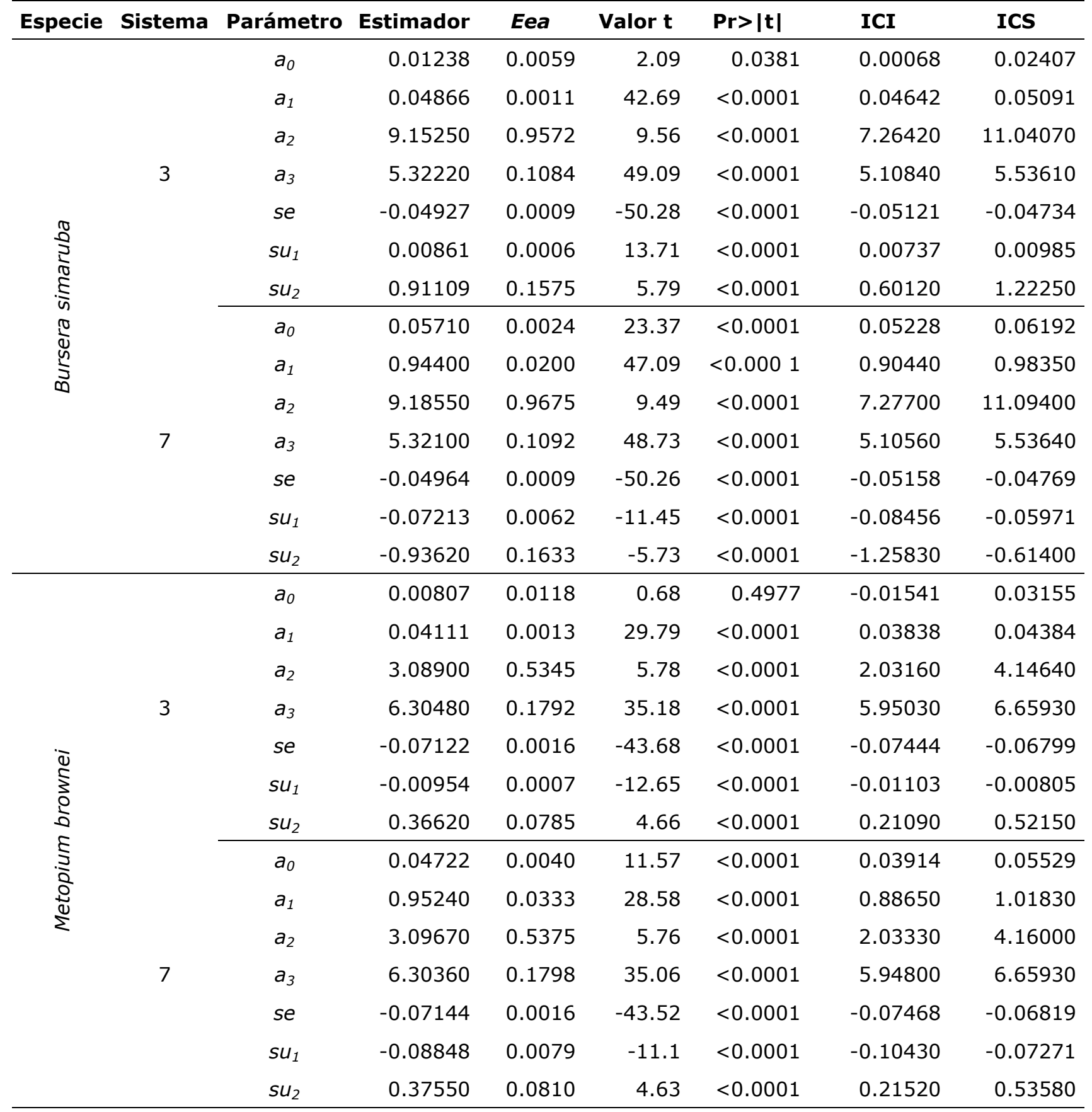

$a_{0}, a_{1}, a_{2}$ y $a_{3}=$ Parámetros de efectos fijos; $s u_{1}$ y $s u_{2}=$ Desviación estándar de los efectos aleatorios; Eea = Error estándar del estimador; se= Desviación estándar de los residuos en el sistema; ICI e ICS = Intervalos de confianza del estimados inferior y superior, respectivamente. 
Para las dos especies, la combinación en la estructura matemática que contiene el modelo de volumen fustal de Spurr en su forma exponencial registró los mayores valores estadísticos en los indicadores de Verosimilitud -2Log y CIAC; por ello las estructuras del modelo 7 fueron las seleccionadas para Bursera simaruba y Metopium brownei, respectivamente. Al contrastar estos valores con los obtenidos al ajustar el modelo general por mínimos cuadrados ordinarios (MCO), se observó una ganancia estadística promedio al aplicar el enfoque de modelos de efectos mixtos de 5.6 \% y $6.9 \%$ para Bursera simaruba y Metopium brownei, respectivamente (Cuadro 4). Además, las pruebas de White para ambas especies resultaron no significativas $(a<$ 0.05), lo cual indicó que los residuales de ambos modelos se distribuyen de manera homocedástica; por lo tanto, se acepta la hipótesis nula $(\mathrm{Ho})$ y se rechaza $\mathrm{Ha}$.

Cuadro 4. Indicadores de ajuste estadístico del modelo de volumen comercial ajustado para Bursera simaruba (L.) Sarg. y Metopium brownei (Jacq.) Urb.

\begin{tabular}{lcccccc}
\hline \multicolumn{1}{c}{ Especie } & \multicolumn{3}{c}{ Bursera simaruba } & \multicolumn{3}{c}{ Metopium brownei } \\
\hline $\begin{array}{c}\text { Modelo con efectos } \\
\text { aleatorios }\end{array}$ & $\begin{array}{l}\text { Modelo } \\
\text { general }\end{array}$ & $\mathbf{3}$ & $\mathbf{7}$ & $\begin{array}{c}\text { Modelo } \\
\text { general }\end{array}$ & $\mathbf{1 1}$ & $\mathbf{1 5}$ \\
\hline Verosimilitud -2 Log & -4617 & -4368 & -4360 & -2588 & -2409 & -2396 \\
CIAC & -4601 & -4354 & -4346 & -2543 & -2395 & -2382 \\
\hline
\end{tabular}

$C I A C=$ Criterio de Información de Akaike Corregido. 
Los sesgos estimados para las dos especies evidenciaron buena precisión en las estimaciones al evaluarlos de forma individual para $V f$ y $R$, así como un modelo completo para estimar el $V c$, ya que en todos los casos fueron menores a $0.03 \mathrm{~m}^{3}$ (Cuadro 5). Además, cuando se hizo la evaluación gráfica y mediante una regresión de los valores observados contra los predichos por variable de interés, no se observaron problemas evidentes en las estimaciones; situación que se reflejó en el valor del coeficiente de determinación $\left(R^{2}\right)$, que en todos los casos fue mayor a 0.9 (Figura 1).

Cuadro 5. Sesgo estimado por variable independiente en el modelo de volumen comercial ajustado.

\begin{tabular}{lccc}
\hline \multicolumn{1}{c}{ Variable independiente } & $\boldsymbol{V f}\left(\mathbf{m}^{\mathbf{3}}\right)$ & $\boldsymbol{R}\left(\boldsymbol{V}_{\boldsymbol{i}} / \boldsymbol{V f}\right)$ & $\boldsymbol{V c}\left(\mathbf{m}^{\mathbf{3}}\right)$ \\
\hline Modelo con la inclusión de efectos aleatorios & Sesgo $(\boldsymbol{E})$ & \\
\hline 7. Bursera simaruba (L.) Sarg. & -0.012543 & 0.029831 & -0.001384 \\
& $\left(R^{2}=0.95\right)$ & $\left(R^{2}=0.93\right)$ & $\left(R^{2}=0.94\right)$ \\
\hline 7. Metopium brownie (Jacq.) Urb. & 0.014684 & 0.019924 & 0.008979 \\
& $\left(R^{2}=0.93\right)$ & $\left(R^{2}=0.94\right)$ & $\left(R^{2}=0.92\right)$ \\
\hline
\end{tabular}

$V f=$ Volumen fustal; $R=$ Razón de volumen basado en el diámetro; $V i=$ Volumen a distintas secciones sobre el fuste; $V c=$ Volumen comercial; $R^{2}=$ Coeficiente de determinación de los valores observados contra los valores predichos. 

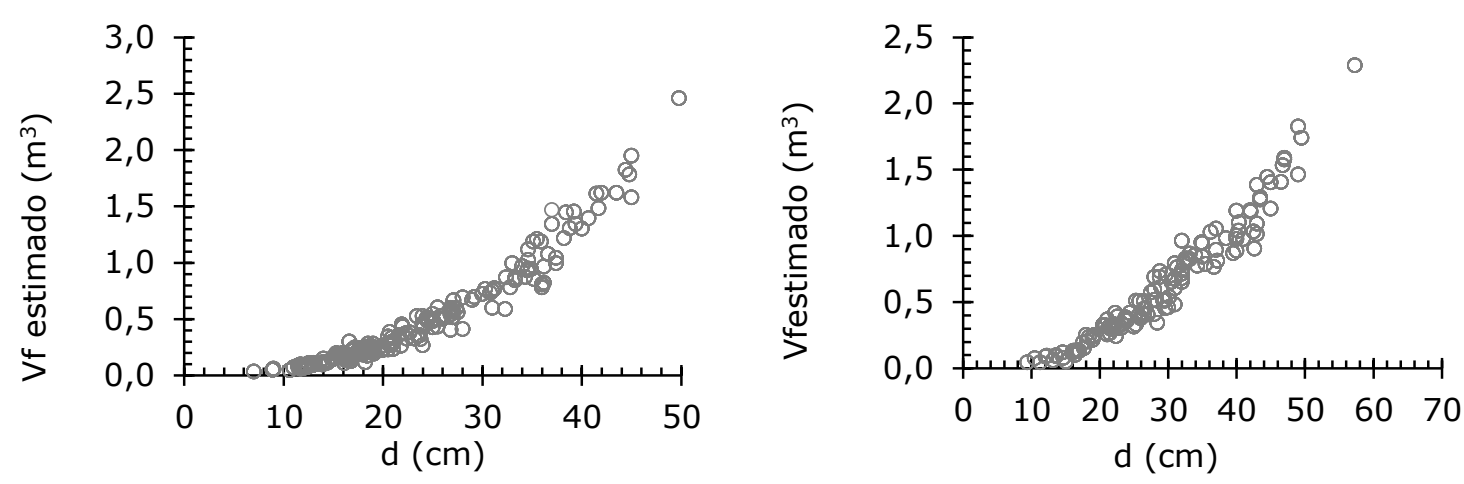

(a)
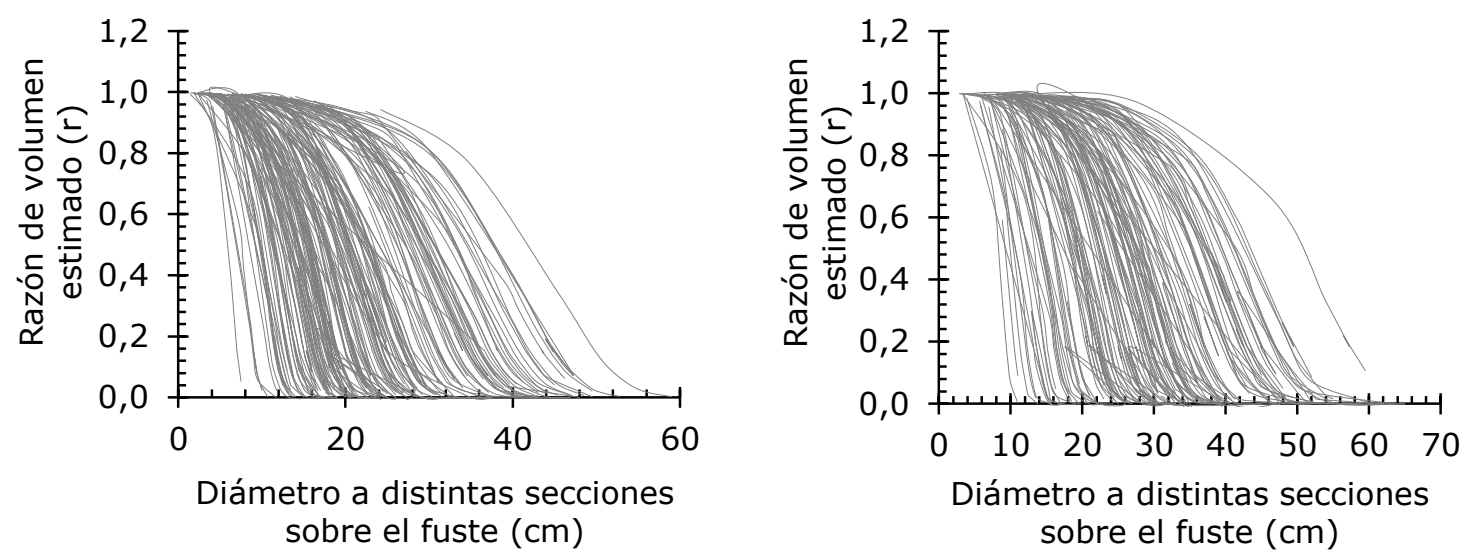

(b)
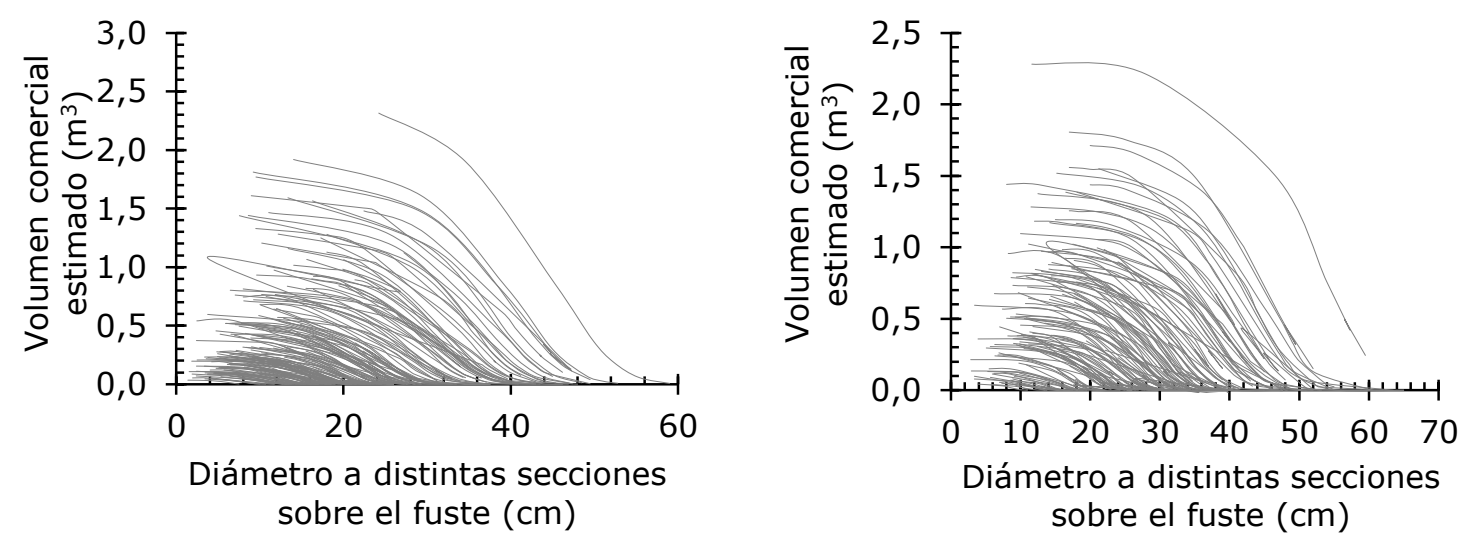

(c)

Figura 1. Tendencia de las estimaciones de volumen fustal, razón de volumen y volumen comercial para las especies de Bursera simaruba (L.) Sarg. (1a, 1b y 1c) y Metopium brownei (Jacq.) Urb. (1d, 1e y $1 \mathrm{f})$. 
Para ejemplificar el uso de la estructura que mejor se ajustó a los datos observados del modelo de volumen comercial, se consideraron como referencia los valores de diámetro normal $(d)=30 \mathrm{~cm}$ y altura total $(H)=15 \mathrm{~m}$ para Bursera simaruba, y $d$ $=40 \mathrm{~cm}$ y $H=20 \mathrm{~m}$ para Metopium brownei, y se usó el modelo 7 con sus parámetros para cada especie para realizar la estimación de volumen fustal $(V f)$ y razón de volumen $(R)$ al emplear por separado las dos secciones del modelo, y al aplicar el modelo completo ajustado para volumen comercial $(V c)$. De la expresión general del modelo se utiliza el primer componente correspondiente a $V f$, el cual al sustituirlo se tiene para Bursera simaruba: Vf $=0.0571\left(\left(30^{2} \cdot 15\right) / 1000\right)^{0.9440}=$ $0.6663 \mathrm{~m}^{3}$, y para Metopium brownei: $V f=0.0472\left(\left(40^{2} \cdot 20\right) / 1000\right)^{0.9524}=1.5049 \mathrm{~m}^{3}$. A continuación, se establece el diámetro mínimo para productos primarios, que en este caso fue $20 \mathrm{~cm}$; enseguida se toma la segunda sección del modelo general y se sustituyen los valores, de lo que resulta para Bursera simaruba:

$R=\operatorname{Exp}(-9.1855 \cdot((20 / 30) / 1000) \cdot \operatorname{Exp}(5.321 *(20 / 30)))=0.8085$, y para Metopium brownei: $R=\operatorname{Exp}(-3.0967 \cdot((20 / 40) / 1000) \cdot \operatorname{Exp}(6.3067 *(20 / 30)))=0.9644$.

Finalmente, para obtener el $V c$ al diámetro mínimo de corta establecido se multiplican los valores de $V f$ y $R$; o bien, se aplica de forma directa el modelo propuesto para cada especie, de la forma siguiente para Bursera simaruba: $V c=$ $\left[0.0571\left(\left(30^{2} \cdot 15\right) / 1000\right)^{0.9440}\right] \cdot\left[\operatorname{Exp}\left(-9.1855 \cdot((20 / 30) / 1000) \cdot \operatorname{Exp}\left(5.321^{*}(20 / 30)\right)\right)\right]=0.5387 \mathrm{~m}^{3}, \mathrm{y}$ para M. brownei: $\left.\quad V c=\left[\begin{array}{lll}0.0472 & \left(\left(40^{2} .\right.\right. & 20) / 1000\end{array}\right)^{0.9524}\right] \quad[\operatorname{Exp}(-$ $3.0967 \cdot((20 / 40) / 1000) \cdot \operatorname{Exp}(6.3067 *(20 / 30)))]=1.4514 \mathrm{~m}^{3}$.

\section{Discusión}

Los MEM permiten corregir la estructura de varianzas-covarianzas asociadas con las variables de clasificación de los árboles (Littell et al., 2006), ya que al agrupar la información en clase, bajo el enfoque de MEM, se reduce el error; puesto que, la variabilidad entre las clases se explica mediante la inclusión de parámetros 
aleatorios, que se estiman de forma simultánea a los parámetros fijos (Castedo et al., 2006; Corral et al. 2019). En esta técnica de análisis (MEM) se asume que la varianza es específica para cada nivel de clasificación (Seoane, 2014), por lo que se logra incrementar el $R^{2}$ y mejorar otros criterios de bondad de ajuste, tales como el AIC y BIC.

En los modelos evaluados, los efectos aleatorios se incluyeron para considerar en las ecuaciones el efecto de las covariables de clasificación durante el ajuste. En este caso, el efecto aleatorio fue específico a cada nivel del factor de clasificación, lo que de acuerdo con De los Santos et al. (2006), Tamarit-Urias et al. (2014) y GarcíaEspinoza et al. (2019) permite la obtención de estimadores más eficientes, precisos y confiables de los parámetros fijos del modelo y la predicción de parámetros aleatorios específicos de cada unidad muestral, que reflejan el patrón de desviación con respecto de la media; pero posterior al ajuste, los valores que resultan para los parámetros aleatorios no se usan para construir las predicciones, por lo que la parsimonia de los modelos no se modifica.

En el caso de la estructura del modelo de volumen 3 para Bursera simaruba, y 7 para M. brownei, todos los parámetros fueron significativos ( $P \geq 0.05)$ (Cuadro 3), similares a lo citado por Quevedo et al. (2010), dichos autores analizaron los modelos de volumen fustal bajo el enfoque de efectos mixtos para Tabebuia rosea Bertol. en Venezuela, donde se realizó una agrupación por categoría diamétrica y transformación de la variable dependiente. De igual forma al estudio realizado por Gómez-García et al. (2016), quienes desarrollaron una función de perfil mediante efectos mixtos para Pinus sylvestris L. en Turquía, la que se ajustó por el método FIRO (first-order), del programa $R$-project, que es el mismo método empleado en este trabajo.

Para la elección del modelo 3 y 7 del Cuadro 1 para Bursera simaruba y Metopium brownei, respectivamente se considera como referencia lo descrito por García y Rapelli (2011), quienes refirieron que los modelos con los valores más cercanos a 
cero en los indicadores de Verosimilitud -2Log y CIAC tienen una mayor verosimilitud en el ajuste. Además, dichos valores fueron congruentes con los criterios de evaluación utilizados por Gómez-García et al. (2016); y por QuiñonezBarraza et al. (2014), autores que ajustaron sistemas compatibles de ahusamientovolumen comercial para las principales especies de Pinus de Durango, México.

Los valores del sesgo en los modelos propuestos por especie y los coeficientes de determinación $\left(R^{2}\right)$ que se obtuvieron al contrastar los datos observados, contra los predichos por variable de interés $(V f, R$ y $V c$ ) coinciden con lo indicado por Pece (1994), quien usó un modelo de razón de volumen para estimar el volumen comercial en Eucalyptus pellita F. Muell. en Brasil, cuyos sesgos al realizar los cálculos por categoría diamétrica fueron entre 0.06 y 0.11 . Además, es similar a lo determinado por Hernández-Ramos et al. (2017), autores que proponen sistemas de razón de volumen para Eucalyptus urophylla en Tabasco y obtienen estimaciones precisas con sesgos menores en las variables de $V t, R$ y $V c$ que los estimados en el presente trabajo (< 0.0077); y $R^{2}$ al contrastar los valores estimados versus observados mayores de 0.94 .

De igual manera, la precisión de las estimaciones de este estudio para el volumen comercial (Vc) son semejantes a lo señalado por Hernández-Ramos et al. (2018b) mediante sistemas de razón de volumen en árboles de Swietenia macrophylla en Quintana Roo, cuyos sesgos para estructuras de razón de volumen basadas en el diámetro normal y en la altura total son de -0.0121 y 0.0053 , respectivamente. Sin embargo, difieren con García-Espinoza et al. (2018) quienes proponen estructuras basadas en la razón de volumen para $P$. pseudostrobus en Michoacán, y registran buena precisión en las estimaciones y un sesgo menor en el $V c$, en comparación con las determinadas en el trabajo que aquí se documenta $\left(<0.001 \mathrm{~m}^{3}\right)$.

La evaluación gráfica de las tendencias en las estimaciones (Figura 1) concuerda con lo registrado por Tapia y Návar (2011) quienes ajustaron modelos de volumen 
fustal y ahusamiento para $P$. pseudostrobus en Nuevo León; Tamarit-Urias et al. (2016), al modelar el perfil fustal de nueve especies del género Pinus y obtener proyecciones acordes a las tendencias de los datos observados. También coincide con los resultados de Tamarit et al. (2017) en la construcción de un sistema de cubicación de árboles individuales para el género Quercus en Puebla y con los de Silva et al. (2018) referentes a las funciones compatibles de ahusamiento y volumen comercial para tres especies de Pinus en Chihuahua.

Las herramientas generadas en el presente trabajo pueden complementarse con las ecuaciones propuestas por García et al. (2017), quienes estiman el volumen fustal a partir de las dimensiones del diámetro de tocón en Bursera simaruba y Metopium brownei en los bosques tropicales de Quintana Roo, y pueden considerarse como una actualización de las ecuaciones propuestas por De los Santos (1976) y Patiño et al. (1994) para ambas especies.

\section{Conclusiones}

Los modelos de volumen propuestos basados en el diámetro a distintas alturas sobre el fuste son confiables para estimar el volumen fustal, ya que emplean la primera parte de la ecuación; la razón de volumen a partir de la segunda parte; y el volumen comercial cuando se utiliza de forma conjunta en las dos especies estudiadas. Lo anterior es una ventaja muy significativa, si se compara con las ecuaciones generadas hace más de 30 años para estimar el volumen de fuste limpio o volumen fuste total para árboles de grandes dimensiones, lo cual no corresponde a los diámetros que se aprovechan hoy en día. Con las estructuras matemáticas propuestas por taxón, es factible construir tablas de distribución de productos para proyectar los rendimientos maderables de áreas bajo aprovechamiento forestal, acorde a las condiciones actuales de Bursera simaruba (chakáh) y Metopium 
brownei (chechem negro) en bosques tropicales localizados en el centro y sur del estado de Quintana Roo, México.

Es necesario realizar este tipo de estudios en Quintana Roo para, al menos, las 30 especies más importantes desde el punto de vista comercial.

\section{Agradecimientos}

Esta investigación fue financiada por el gobierno del estado de Quintana Roo a través del Instituto Forestal del Estado de Qintana Roo (INFOQROO) por medio del proyecto "Estudio para realizar acciones de tablas de volúmenes de ocho especies forestales maderables". SINCOP-INIFAP 232179Y.

\section{Conflicto de intereses}

Los autores declaran no tener conflicto de intereses.

\section{Contribución por autor}

Jonathan Hernández Ramos, Adrián Hernández Ramos y Xavier García Cuevas: diseño del estudio, toma y análisis de datos, ajuste estadístico y elaboración de manuscrito; Juan Carlos Tamarit Urias y Enrique Buendía Rodríguez: revisión, análisis y discusión del documento. 


\section{Referencias}

Balzarini, M., R. Macchiavelli y F. Casanoves. 2005. Aplicaciones de modelos mixtos en agricultura y forestería. Turrialba, Costa Rica: Mimeo: Centro Agronómico Tropical de Investigación y Enseñanza (CATIE). Cartago, Costa Rica. 189 p. https://www.researchgate.net/publication/283491085_Aplicaciones_de_Modelos_Mi xtos_en_Agricultura_y_Forestería (30 de septiembre de 2021).

Barrios, A., A. M. López y V. Nieto. 2014. Predicción transición de volúmenes comerciales de Eucalipto grandis a través de modelos de volumen total y de razón. Colombia Forestal 17: 137-149. Doi: 10.14483/udistrital.jour.colomb.for.2014.2.a01. Beal, S. L. and L. B. Sheiner. 1982. Estimating Population Kinetics. CRC Critical Reviews in Biomedical Engineering 8: 195-222.

Burkhart, H. E. 1977. Cubic-foot volume of Loblolly Pine to any merchantable top limit. Southern Journal of Applied Forestry 1(2): 7-9. https://www.researchgate.net/publication/233704988_CubicFoot_Volume_of_Loblolly_Pine_to_Any_Merchantable_Top_Limi (30 de septiembre de 2021). Carrero, O., M. Jerez, R. Macchiavelli, G. Orlandoni y J. Stock. 2008. Ajuste de curvas de índice de sitio mediante modelos mixtos para plantaciones de Eucalyptus urophylla en Venezuela. Revista Interciencia 33(4): 265-272. https://www.researchgate.net/publication/46406313 (30 de septiembre de 2021). Castedo, D. F., U. Diéguez A., M. Barrio A., M. Sánchez R. and K. V. Gadow. 2006. A generalized height-diameter model including random components for radiata pine plantations in northwestern Spain. Forest Ecology and Management 229: 202-213. Doi: 10.1016/j.foreco.2006.04.028.

Corral-Rivas, S. y J. J. Návar-Cháidez. 2009. Comparación de técnicas de estimación de volumen fustal total para cinco especies de pino de Durango, México. Revista Chapingo Serie Ciencias Forestales y del Ambiente 15(1): 5-13. http://www.scielo.org.mx/pdf/rcscfa/v15n1/v15n1a1.pdf (30 de septiembre de 2021). 
Corral-Rivas, S., J. G. Álvarez-González, F. Crecente-Campo and J. J. Corral-Rivas. 2014. Local and generalized height-diameter models with random parameters for mixed, uneven-aged forests in Northwestern Durango, Mexico. Forest Ecosystems 1: 1-9. https://forestecosyst.springeropen.com/track/pdf/10.1186/2197-5620-16.pdf (30 de septiembre de 2021).

Corral R., S., A. M. Silva A. y G. Quiñonez B. 2019. Modelo generalizado no-lineal altura-diámetro con efectos mixtos para siete especies de Pinus en Durango, México. Revista Mexicana de Ciencias Forestales 10(53): 86-117. Doi: 10.1186/2197-5620-1-6.

De los Santos, V., M. 1976. Tablas de volúmenes para montes de la Península de Yucatán. Escuela Nacional de Agricultura. Departamento de Enseñanza, Investigación y Servicio en Bosques. Texcoco de Mora, Edo. de Méx., México. 82 p. De los Santos P., H. M., M. Montero M. y M. Kanninen. 2006. Curvas dinámicas de crecimiento en altura dominante para Terminalia amazonia (Gmel.) Excell en Costa Rica. Agrociencia 40: 521-532. http://www.colpos.mx/agrocien/Bimestral/2006/julago/art-11.pdf (30 de septiembre de 2021).

Diéguez-Aranda, U., A. Rojo, F. Castedo-Dorado, J. G. Álvarez, M. Barrio-Anta, F. Crecente-Campo, J. M. González G., C. Pérez-Cruzado, R. Rodríguez S., C. A. López-Sánchez, M. Á. Balboa-Murias, J. J. Gorgoso V. y F. Sánchez R. 2009. Herramientas selvícolas para la gestión forestal sostenible en Galicia. Universidad de Santiago de Compostela. Galicia, España. 259 p. https://www.researchgate.net/publication/305443573_Herramientas_selvicolas_par a_la_gestion_forestal_sostenible_en_Galicia (30 de septiembre de 2021).

Gallegos, R., A., M. Sánchez D., G. A. González C., L. Román M., E. Hernández A. y A. Mora S. 2012. Diagnóstico del potencial, productividad y manejo de especies nativas maderables tropicales con alto potencial comercial. Guadalajara, Jalisco, México: Universidad de Guadalajara-Centro Universitario de Ciencias Biológicas y 
Agropecuarias. Guadalajara, Jal., México. 41 p.

https://www.itto.int/files/itto_project_db_input/2596/Technical/Capitulo\%202\%20D iagn\%C3\%B3stico\%20del\%20potencial,\%20productividad\%20y\%20manejo\%20de $\% 20$ especies\%20nativas\%20maderables.pdf (30 de septiembre de 2021).

García C., X., J. Hernández R., A. Hernández R., G. Quiñonez B., J. C. Tamarit U. y G. G. García E. 2017. Predicción del diámetro normal, altura y volumen a partir del diámetro del tocón en especies tropicales. Revista Mexicana de Ciencias Forestales 8(43): 89-116. Doi: 10.29298/rmcf.v8i43.67.

García, M. del C. y C. Rapelli. 2011. Selección de un modelo lineal mixto de niveles múltiples para modelos el crecimiento de la soja. Revista FABICIB 15: 11-22.

Doi: 10.14409/fabicib.v15i1.877.

García-Espinoza, G. G., O. A. Aguirre-Calderón, G. Quiñonez-Barraza, E. A. AlanísRodríguez, H. M. De los Santos-Posadas and J. J. García-Magaña. 2018. Taper and volume systems based on ratio equations for Pinus pseudostrobus Lindley in Mexico. Forests 9(344): 1-14. Doi:10.3390/f9060344.

García-Espinoza, G. G., O. A. Aguirre-Calderón, G. Quiñonez-Barraza, E. AlanísRodríguez, M. A. González-Tagle and J. J. García-Magaña. 2019. Global-local and fixed-random parameters to model dominant height growth of Pinus pseudostrobus Lindley. Revista Chapingo Serie Ciencias Forestales y del Ambiente 25(1): 141-156. Doi: $10.5154 /$ r.rchscfa.2018.06.047.

Gómez-García, E., U. Diéguez-Aranda, R. Özçelik, M. Sal-Cando, F. Castedo-Dorado, F. Crecente-Campo, J. J. Corral-Rivas y M. Arias-Rodil. 2016. Desarrollo de una función de perfil mediante modelos mixtos para Pinus sylvestris en Turquía: Selección de parámetros fijos a expandir. Bosque 37(1): 159-167. Doi: $10.4067 /$ S0717-92002016000100015. Herbario CICY. 2010. Flora Digital: Península de Yucatán. https://www.cicy.mx/sitios/flora\%20digital/indice_busqueda.php (13 de marzo de 2020). 
Hernández-Ramos, J., H. M. De los Santos-Posadas, J. R. Valdéz-Lazalde, J. C. Tamarit-Urias, G. Ángeles-Pérez, A. Hernández-Ramos, B. Méndez-López y A. Peduzzi. 2017. Estimación del volumen comercial en plantaciones de Eucalyptus urophylla con modelos de volumen total y de razón. Agrociencia 51: 561-580. http://www.scielo.org.mx/pdf/agro/v51n5/1405-3195-agro-51-05-00561.pdf (30 de marzo de 2021).

Hernández-Ramos, J., A. Hernández-Ramos, X. García-Cuevas, J. C. Tamarit-Urias, L. Martínez-Ángel y J. J. García-Magaña. 2018a. Ecuaciones de volumen total y de razón para estimar el volumen comercial de Swietenia macrophylla King. Colombia Forestal 21(1): 34-46. Doi:10.14483/2256201X.11965.

Hernández-Ramos, J., A. Hernández R., X. García C., L. Martínez Á., J. C. Tamarit U. y G. G. García E. 2018b. Sistema compatible de ahusamiento-volumen comercial para Swietenia macrophylla King (caoba) en Quintana Roo, México. Madera y Bosques. 24(3): 1-11. Doi: 10.21829/myb.2018.2431441.

Littell, R., G. Milliken, W. Stroup, R. Wolfinger and O. Schabenberger. 2006. SAS for mixed models. SAS Institute Inc. Cary, NC, USA. 795 p. Doi: 10.1111/j.15410420.2006.00596_6.x.

López M., J. O., B. Vargas L., O. A. Aguirre C., , C. G. Aguirre C., P. A. Macario M., M. Martínez S. and J. G. Álvarez G. 2019. Compatible taper-volume systems for major tropical species in Mexico. Forestry 00: 1-19. Doi:10.1093/forestry/cpz033.

Martínez G, M. A., V. A. Sánchez y F. J. Faulin. 2006. Bioestadística amigable. Ediciones Díaz de Santos. Barcelona, España. 936 p.

Niño L., G. S., P. A. Ramos M., A. Barrios y A. M. López A. 2018. Modelos compatibles de ahusamiento-volumen para árboles de Gmelina arborea Roxb. en el Alto Magdalena, Colombia. Colombia Forestal 21(2): 174-187.

Doi: $10.14483 / 2256201 X .12249$. 
Organización Internacional de Maderas Tropicales (OIMT). 2020. ITTO Lesser used species. http://www.tropicaltimber.info/ (13 de marzo de 2020).

Özçelik, R. and M. Göçeri. 2015. Compatible merchantable stem volume and taper equations for Eucalyptus plantations in the Eastern Mediterranean Region of Turkey. Turkish Journal of Agriculture and Forestry, 39: 851-863. Doi:10.3906/tar-1501-27.

Patiño V., F., J. L. López T. y A. Gómez D. 1994. Selva (Versión 4) Paquete de cómputo para procesar datos de inventarios forestales para especies de la Península de Yucatán. Mérida, Yucatán: Instituto Nacional de Investigaciones Forestales y Agropecuarias. Mérida, Yuc., México. 46 p.

Pece R., M. 1994. Tabla de volumen comercial para Eucalyptus pellita utilizando el método de la razón volumétrica. Quebracho 2: 54-63. https://fcf.unse.edu.ar/archivos/quebracho/q2_07.pdf (30 de septiembre de 2021).

Pinheiro, J. C. and D. M. Bates. 1995. Approximations to the Log-Likelihood Function in the nonlinear mixed-effects model. Journal of Computational and Graphical Statistics 4: 12-35.

https://www.jstor.org/stable/1390625?seq=2\#metadata_info_tab_contents (30 de septiembre de 2021).

Prodan, M., R. Peters, F. Cox y P. Real. 1997. Mensura Forestal.: Instituto Interamericano de Cooperación para la Agricultura (IICA)/BMZ/GTZ. San José, Costa Rica. 561 p.

Quevedo, A., M. Jerez, A. Y. Moret y O. Noriega. 2010. Ecuaciones de volumen y calidad de forma para Tabebuia rosea (Bertol.) en plantaciones en líneas. Barinas, Venezuela. Revista Forestal Venezolana 54(2): 195-205.

http://www.ula.ve/ciencias-forestales-ambientales/indefor/wp- 
content/uploads/sites/9/2016/11/2010_Quevedo_et_al.pdf (30 de septiembre de 2021).

Quiñonez B., G., Cruz C., F., Vargas L., B. y Hernández, F. J. 2012. Estimación del diámetro, altura y volumen a partir del tocón para especies forestales de Durango. Revista Mexicana de Ciencias Forestales 3(9): 23-39. Doi:10.29298/rmcf.v3i9. Quiñonez-Barraza, G., H. M. De los Santos-Posadas, J. G. Álvarez-González y A. Velázquez-Martínez. 2014. Sistema compatible de ahusamiento y volumen comercial para las principales especies de Pinus en Durango, México. Agrociencia 48: 553-567. http://www.scielo.org.mx/scielo.php?script=sci_arttext\&pid=S140531952014000500008 (30 de septiembre de 2021).

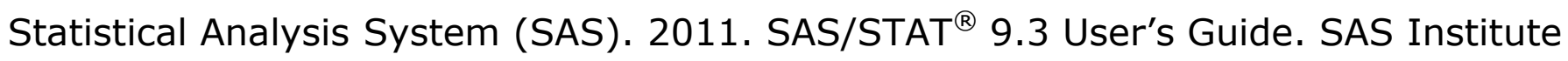
Inc. Cary, NC, USA. 106 p.

Román M., M. L., A. Mora S. y G. A. González C. 2016. Sistemas agroforestales con especies de importancia maderable y no maderable, en el trópico seco de México. Avances en Investigación Agropecuaria 20(2): 53-72. http://ww.ucol.mx/revaia/portal/pdf/2016/mayo/5.pdf (30 de septiembre de 2021). Schabenberger, O. and F. Pierce. 2002. Contemporary statistical models for the plant and soil sciences. CRC Press. Boca Raton, FL, USA. 730 p.

Secretaria del Medio Ambiente y Recursos Naturales (Semarnat). 2014. Inventario Estatal Forestal y de Suelos - Quintana Roo 2013. Colección de inventarios estatales forestales y de suelos 2013-2014.: Editorial Prometeo. Guadalajara, Jal., México. 125 p.

Secretaria del Medio Ambiente y Recursos Naturales (Semarnat). 2017. Anuario estadístico de la producción forestal 2016. México, D. F., México. https://www.gob.mx/cms/uploads/attachment/file/282951/2016.pdf (30 de septiembre de 2021). 
Seoane, J. 2014. ¿Modelos mixtos (lineales)? Una introducción para el usuario temeroso. Etologuía 24: 15-37. https://pdfslide.net/download/link/modelos-mixtoslineales-una-introduccion-para-el-etologuia-24-2014 (30 de septiembre de 2021).

Silva G., E., M. A. Nava M., F. J. Hernández y J. G. Colín. 2018. Funciones compatibles de ahusamiento-volumen para tres especies de Pinus en la Unidad de Manejo Forestal 0808 del estado de Chihuahua. Investigación y Ciencia de la Universidad Autónoma de Aguascalientes 26(73): 58-67.

Doi: 10.33064/iycuaa201873207.

Silva J., A., F. J. Fuentes, H. Georg, A. Gallegos, P. S. Rosa M., M. Flores y J. G. Hernández R. 2011. Industrialización, comercialización y manejo sostenible de diez especies nativas mexicanas.: Universidad de Guadalajara. Centro Universitario de Ciencias Biológicas y Agropecuarias. Guadalajara, Jal., México 37 p.

Spurr, S. H. 1952. Forest inventory. John Wiley and Sons. New York, NY, USA. 472 p. Tamarit-Urias, J. C., H. M. De los Santos-Posadas, A. Aldrete, J. R. Valdez-Lazalde, H. Ramírez-Maldonado y V. Guerra-De la Cruz. 2014. Ecuaciones dinámicas de índice de sitio para Tectona grandis en Campeche, México. Agrociencia 48(2): 225-238. http://www.scielo.org.mx/pdf/agro/v48n2/v48n2a8.pdf (30 de septiembre de 2021).

Tamarit U., J. C., G. Quiñonez B., C. Ordoñez P. J. C. Monárrez G. y E. Rojas D. 2016. Modelos de ahusamiento para especies de coníferas en Puebla. INIFAP-CIRGOC-Campo Experimental San Martinito. Puebla, Pue., México. Folleto Técnico Núm. 85. 20 p.

Tamarit U., J. C., E. Rojas D., G. Quiñonez B., C. Ordoñez P. y J. C. Monárrez G. 2017. Sistema de cubicación para árboles individuales de Quercus sp. en bosques bajo manejo de Puebla, México. Revista Mexicana de Ciencias Forestales 8(40): 726. Doi:10.29298/rmcf.v8i40.37. 
Tapia, J. y J. Návar. 2011. Ajuste de modelos de volumen y funciones de ahusamiento para Pinus pseudostrobus Lindl. en bosques de pino de la sierra madre oriental de Nuevo León, México. Foresta Veracruzana 13(2): 19-28. https://www.redalyc.org/pdf/497/49721457004.pdf (30 de septiembre de 2021).

Todos los textos publicados por la Revista Mexicana de Ciencias Forestales - sin excepciónse distribuyen amparados bajo la licencia Creative Commons 4.0 Atribución-No Comercial (CC BY-NC 4.0 Internacional), que permite a terceros utilizar lo publicado siempre que mencionen la autoría del trabajo y a la primera publicación en esta revista. 